FZMw Jg. 6 (2003) S. 189-207

\title{
Musikwissenschaftliche Forschungsansätze auf fremdem Terrain: Musiktheater als "Regietheater"
}

\author{
von Kordula Knaus
}

$-1-$

\section{Musiktheater und Interdisziplinarität}

Musiktheater ist interdisziplinär. An dieser Feststellung kann es schon deshalb keinen Zweifel geben, weil die Begriffsbildung "Musiktheater" bereits zwei Disziplinen in sich vereint, nämlich Musik einerseits und Theater andererseits. Wenn Musiktheater interdisziplinär ist, gilt dies dann ohne weiteres auch für die Musiktheaterforschung? Die Antwort lautet: Nein - das zeigt uns die Forschungspraxis oft genug. Aber Musiktheaterforschung kann und sollte es dennoch vermehrt sein.

In der gegenwärtigen Musiktheaterforschung finden sich kaum Annäherungen oder Anknüpfungspunkte an die aktuelle Bühnenpraxis. Was auf einer Theaterbühne stattfindet, ist höchstens als historischer Forschungsansatz interessant, als quasi szenische Aufführungspraxis parallel zur musikalischen. Solche Forschungsansätze wären etwa die Untersuchung der Bühnenmaschinerie der Opera seria oder die Auswertung erhaltener Regiebücher. Wie jedoch die Theaterpraxis darüber hinaus mit ihrer Vielzahl an szenischen Realisierungsmöglichkeiten für die Musiktheaterforschung nutzbar gemacht werden kann, soll im Folgenden dargestellt werden.

$$
-3-
$$

Die Musikwissenschaft als Disziplin gewinnt heute immer neue Interpretationen der etwa dreißig Repertoireopern, dem so genannten (hier allerdings nur auf die Oper bezogenen) musikalischen Kanon. Genau dieses Repertoire findet sich auch auf den Spielplänen der Opernbühnen. Das heißt, dass der Gegenstand sowohl der Wissenschaft als auch der Bühne nahezu identisch ist. Dabei bleiben in der Wissenschaft die neuen faktischen Erkenntnisse bei der Untersuchung des Opernrepertoires gering - das Material ist quasi aufgearbeitet. Trotzdem nehmen Publikationen über Opern von Mozart 
oder Wagner kein Ende. Die Musiktheaterwissenschaft gewinnt ihre Erkenntnisse hierbei aus der neuen Interpretation des vorhandenen Materials - aus neuen Zugängen, neuen Methoden, neuen Gedankengängen, neuen Kontextualisierungen. Diese ständigen Änderungen des Betrachtungswinkels sind ein Phänomen jeder Forschung, die unweigerlich zeitbedingt ist. "Regietheater" - wenn der Begriff vorerst ganz allgemein als ein szenisches Theatererlebnis, das eine interpretatorische Ebene eröffnet, aufgefasst wird - tut und ist nichts anderes. Es sucht nach neuen Wegen der Auslegung des Opernrepertoires, nach neuen Formen der Darstellung. Noch offensichtlicher als die Wissenschaft ist die Bühne dabei Spiegel der Zeit.

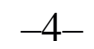

Diese Parallelerscheinung kann für einen interdisziplinären Forschungsansatz nutzbar gemacht werden, etwa wenn in der Musikwissenschaft eine Auseinandersetzung mit den szenischen Visionen der jüngsten Vergangenheit und der Gegenwart erfolgt. Die Inszenierung, als Einbringung des theatralischen Aspektes des Musiktheaters, kann und sollte dabei das zu oft vernachlässigte Objekt wissenschaftlicher Forschung sein. Doch wie kann wissenschaftlich an eine Operninszenierung herangegangen werden? Was sind die methodischen Probleme, die gelöst werden müssen, und wie sehen die Lösungen dieser Schwierigkeiten letztlich aus?

$$
-5-
$$

\section{Regietheater als Forschungsobjekt}

Ein erster Schritt in der Untersuchung einer Inszenierung muss die Darstellung und Beschreibung des szenischen Ereignisses an sich sein ${ }^{1}$. Dabei gibt es für die Forscherin oder den Forscher nur zwei Möglichkeiten, sich eine Inszenierung anzueignen - durch eigenes Beiwohnen einer Aufführung im Theater oder durch Sichtung von Sekundärmaterialien wie Video- und Fernsehaufzeichnungen, Fotos oder Dias. Für beide Möglichkeiten gilt, dass die Erläuterung szenischer Vorgänge, die durch optische Eindrücke vermittelt werden, immer subjektiv ist und nicht vom Blickwinkel der Betrachterin oder des Betrachters isoliert werden kann. Diese selektive Aufmerksamkeit tritt vor allem im Live-Ereignis der Theateraufführung unabwendbar auf, da diese

Die nachfolgenden Anmerkungen zur Praxis des Theaterbetriebs basieren wesentlich auf meiner mehrjährigen Erfahrung als Regieassistentin und Spielleiterin am Opernhaus Graz, wo ich bedingt durch die internationalen Fluktuationen in der Opernwelt - auch entsprechende Informationen über andere Bühnen sammeln konnte. 
immer in Echt-Zeit abläuft und auch nur so verfolgt werden kann. Eine Verschriftlichung und exakte Beschreibung der szenischen Ereignisse kann aber erst im Nachhinein und aus dem Gedächtnis erfolgen und wird daher der benötigten Exaktheit entbehren. Unweigerlich ist die Wissenschaft folglich auf Reproduktionen des LiveEreignisses in Form von Aufzeichnungen angewiesen, und dies vor allem bei der Behandlung von Inszenierungen, die nicht mehr als reales Theatererlebnis nachvollziehbar sind. Selbst nur einige Jahre zurïckliegende Inszenierungen sind bedingt durch die gegenwärtige Spielplanpolitik der meisten Theater, die eine Inszenierung meist nur für wenige Spielzeiten in ihrem Spielplan vorsieht - einzig noch über technische Medien zugänglich. In einer Videoaufzeichnung jedoch erfolgt durch die verschiedenen Bildeinstellungen bereits eine Selektion, die für die Forschenden vorgegeben ist. Bei älteren Inszenierungen ist zudem meist überhaupt kein Videomaterial verfügbar, so dass zur Untersuchung auf Fotos und bereits erfolgte Beschreibungen zurückgegriffen werden muss. Hier sind szenische Abläufe nur noch aus dritter Hand und in Bildabfolgen nachvollziehbar und hinterlassen dementsprechend ein oberflächlicheres und ungenaueres Bild.

$-6-$

Weiterhin ist zu berücksichtigen, dass sich die Beschreibung von Inszenierungen immer nur auf eine gesehene Aufführung beziehen kann und daher eine Momentaufnahme darstellt. Eine Inszenierung aber ist ein sich entwickelnder Prozess, bei dem sich von Aufführung zu Aufführung szenische Aktionen verändern. Diese Modifikationen hängen zum Großteil von den darstellenden Personen $\mathrm{ab}^{2}$. Selbst bei gleich bleibender Besetzung verschiedener Aufführungen sind Tagesverfassung, das Empfinden für die Zeitlichkeit szenischer Abläufe, die Körperspannung und andere Befindlichkeitsfaktoren der Sängerinnen und Sänger ausschlaggebend für die Originalität jeder Aufführung. (Letztendlich gehen wir gerade wegen dieser Einmaligkeit heute noch ins Theater und konsumieren Musiktheater nicht nur noch über verschiedene elektronische Medien.) Dazu kommt, dass Besetzungen sich in der gängigen Theaterpraxis häufig ändern, was zwangsläufig zu unterschiedlichen szenischen Varianten führt, da verschiedene Personen eine Rolle nicht identisch darstellen können. Außerdem sind Sängerinnen und Sänger meist in ungleicher Weise am Entwicklungsprozess einer Inszenierung beteiligt -

Im Folgenden werden aus dem darstellenden Personal die Gesangssolistinnen und -solisten in den Mittelpunkt gerückt, in dem Bewusstsein, dass ähnliche Bedingungen auch für das Personal des Chores, des Balletts oder der Statistinnen und Statisten gelten. 
dies hängt wiederum einerseits davon ab, ob eine Sängerin oder ein Sänger als Erstoder Zweitbesetzung vorgesehen ist (was in vielen Theatern entweder mit entsprechend zahlreichen Probenmöglichkeiten im ersten Fall oder entsprechend wenigen im zweiten Fall verbunden ist) oder überhaupt für eine Vorstellung, etwa im Krankheitsfall der vorgesehenen Darstellerin oder des Darstellers, lediglich einspringt und im schlimmsten Fall erst am Tag der Aufführung anreist, um sich ein Inszenierungskonzept in kürzester Zeit aneignen zu müssen. Andererseits begründet sich die Dynamik einer Inszenierung auch in der Motivation der Künstlerinnen und Künstler, ihrer Einstellung zum entsprechenden Regiekonzept, ihrer Bühnenwirksamkeit und ihren szenischen Fähigkeiten. Eine Aufführung ist außerdem von technischen Faktoren (etwa von der Präzision der Licht- und Vorhangszeichen, der Nebelmenge, den Verwandlungen auf offener Bühne etc.) sowie musikalischen (Dirigat, Orchester, Bühnenpersonal) beeinflusst. Letztendlich müsste auch das Publikum einer Aufführung hier genannt werden, das durch seine Ge- oder Missfallensbekundungen auf die an der Aufführung Beteiligten einwirkt. Das Untersuchungsobjekt Inszenierung ist somit nie exakt fassbar und lässt immer einen Spielraum für Varianten offen.

$$
-7-
$$

Soll nun in einem zweiten Schritt über die ausschließliche Beschreibung szenischer Ereignisse hinausgegangen werden, so wird sich zwangsläufig die Frage der Bedeutung einzelner szenischer Aktionen stellen. Selbstverständlich wäre der einfachste Weg, um zu einer gesicherten Antwort zu gelangen, jene zu befragen, welche die Inszenierung geplant und realisiert haben. Es muss jedoch davon ausgegangen werden, dass verbale Äußerungen immer nur ein Bruchteil dessen sind, was an intellektuellem Überbau in einer Inszenierung vorhanden ist und im Laufe einer Produktionszeit entsteht. Eine Inszenierung stellt ein optisch-akustisches Produkt dar, dessen Ideenhintergrund oft nur für Interviews oder Programmhefte notgedrungen verbalisiert wird. Die eigentliche gedankliche Arbeit findet entweder im "stillen Kämmerchen" oder in den Köpfen der Beteiligten statt. Und schließlich: Wer sind diese Köpfe? Ist es immer der Regisseur oder die Regisseurin? Was ist der Beitrag des Teams ${ }^{3}$ ? Ist die Intention einer Regisseurin

Das künstlerische Team einer Inszenierung besteht generell aus der szenischen Leitung (Regie, Bühnenbild, Kostüm, Licht), der musikalischen Leitung (Dirigat, Chorleitung), der Dramaturgie und den jeweils dazugehörenden Assistenzen. Sind weitere Kompetenzen für eine Aufführung erforderlich, wird dieses Team entsprechend erweitert, etwa wenn im aufgeführten Werk ein Ballett auftritt, das eine Choreographie benötigt. Die Beiträge der beteiligten Einzelpersonen können recht unterschiedlich gewichtet sein - im Idealfall erarbeiten szenische und musikalische 
oder eines Regisseurs im szenischen Ergebnis überhaupt erkennbar? Denn es kann vorkommen, dass Beschreibungen und Interpretationen der Regisseurinnen und Regisseure in der konkreten Realisierung schlechterdings nicht verifizierbar sind. Dass die Entstehung einer Inszenierung ein in den eben beschriebenen Maßen dynamischer Prozess ist, macht die Isolierung konkreter interpretatorischer Aussagen sehr schwierig.

$$
-8-
$$

Die Schwierigkeiten bei der Fixierung des Forschungsobjektes und der Untersuchung der Sekundärmaterialien können lediglich durch einen sehr bewußten und vorsichtigen Umgang mit den entsprechenden Quellen gehandhabt werden. Erst nachdem diese Vorarbeiten geleistet sind, kann in einem dritten Schritt die eigentliche Arbeit der Musiktheaterwissenschaft beginnen: die Herstellung von Zusammenhängen, Kontextualisierungen und Interpretationen.

$$
-9-
$$

\section{Der Schluss von Richard Wagners Götterdämmerung}

Wie eine solche eben theoretisch beschriebene Untersuchung einer Inszenierung praktisch erfolgen kann, soll nun anhand des Schlusses der Götterdämmerung vorgestellt werden, dem letzten Teil von Richard Wagners Ring des Nibelungen. Der Ring des Nibelungen kann als ein Schlüsselwerk szenischer Interpretation in der Opernliteratur angesehen werden. Seine "ganze Weltanschauung" ${ }^{4}$ wollte Wagner in dieser Tetralogie von drei Abenden samt einem Vorspiel ausdrücken - er verwob sie in beziehungsreiche Dramen zwischen Mythos und Geschichte. Gerade der Schluss des Gesamtwerks scheint dabei von besonderem Interesse zu sein. Wie endet ein Werk, das über vier Abende hinweg vorführt, dass mit Recht und Ordnung lediglich Missbrauch betrieben wird und dass Machtstreben zu Entmenschlichung führt? Welche Konsequenzen werden aus der im Ring geübten umfassenden politischen und gesellschaftlichen Kritik gezogen? Das Ende der Götterdämmerung stellt die Frage nach der Zukunft - der Zukunft der Menschheit.

Leitung gemeinsam mit der Dramaturgie ein Konzept und setzen es dann in den jeweiligen Zuständigkeitsbereichen um (entsprechende Konzeptionsgespräche sind etwa für die RingInszenierung von Ruth Berghaus in Frankfurt, auf die ich noch zurückkommen werde, durch Aufzeichnungen mehrerer Assistenten belegt, Ruth Berghaus-Archiv, Zeuthen, Inv.Nr. 982, 1008, 1024, 1043).

4 Richard Wagner in einem Brief an Theodor Uhlig vom 31. Mai 1852, in: Richard Wagner, Sämtliche Briefe, Bd. 4, hg. von Gertrud Strobel und Werner Wolf, Leipzig u.a. 1979, S. 385. 
Richard Wagners Antwort auf diese Zukunftsfrage ist in der endgültigen Schlussfassung der Götterdämmerung ${ }^{5}$ denkbar vage formuliert - wirkliche Perspektiven sind kaum auszumachen. Göttinnen und Götter gehen in Flammen auf, Wotans Traum der Vereinigung von Brünnhilde und Siegfried zum "Menschenpaar der Zukunft" kann (wenn überhaupt) nur in einer jenseitigen Welt stattfinden, Hagen und Gunther sind ebenfalls tot, das Schicksal Alberichs und Gutrunes scheint ungewiss, und Erda hat sich schon am Beginn des dritten Aktes von Siegfried zu ewigem Schlaf zurückgezogen. Die Rheintöchter bekommen zwar den Ring zurück - da dieser aber nicht wieder in das Rheingold zurïckverwandelt wird, bleibt er als Machtsymbol erhalten und damit der Gefahr erneuter Diebstähle ausgesetzt. Am Rande des Weltuntergangs sehen "Männer und Frauen, in höchster Ergriffenheit dem Feuerschein am Himmel zu" ${ }^{\prime 6}$. Da ihnen die Zusammenhänge rund um die Ereignisse der Ring-Intrige aber verborgen geblieben sind, können sie nichts tun, als staunend der Apokalypse zusehen.

$$
-11-
$$

Scheinen inhaltliche Fakten kaum Rückschlüsse auf mögliche Zukunftsperspektiven zuzulassen, so gibt die musikalische Gestaltung Wagners erst recht Rätsel auf. Das letzte 'Wort' in der Musik der Götterdämmerung fällt dem so genannten "Liebeserlösungsmotiv"7 $^{7} \mathrm{zu}$, das bereits im 3. Aufzug der Walküre zu Sieglindes Worten "O hehrstes Wunder! Herrlichste Maid!" erstmals Verwendung findet und danach erst wieder im Schlussgesang Brünnhildes auftaucht.

\section{Richard Wagner, Götterdämmerung, 3. Aufzug, T. 1594-1595 (1. und 2. Violinen):}

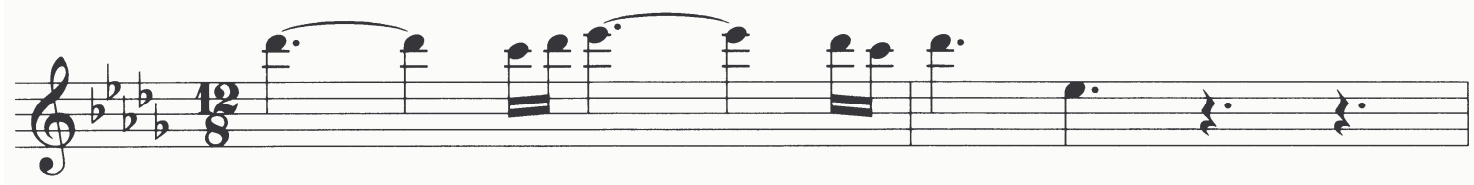

$\mathrm{Zu}$ den anderen Schlussfassungen vgl. Kordula Knaus, "Weißt du was daraus wird?". Szenische Realisierungsmöglichkeiten des Schlusses der "Götterdämmerung" (im Erscheinen).

6 Richard Wagner, Der Ring des Nibelungen. Ein Bühnenfestspiel für drei Tage und einen Vorabend. Dritter Tag: Götterdämmerung. Dritter Aufzug, hg. von Hartmut Fladt, Mainz 1981 (Richard Wagner. Sämtliche Werke, Bd. 13, III), T. 1549ff.

7 Obwohl die Bezeichnung, die durch Hans von Wolzogen (Führer durch die Musik zu Richard Wagner's Festspiel "Der Ring des Nibelungen". Ein thematischer Leitfaden, Leipzig o.J.) geprägt wurde, nicht besonders glücklich gewählt ist, wird sie im Folgenden beibehalten - in dem Bewusstsein, dass solche Bezeichnungen keine verbindliche semantische Klassifizierung darstellen. 
Das Motiv ist in der Walküre mit verschiedenen Bedeutungsebenen verknüpft: Es ist einerseits Ausdruck von Sieglindes Willen, weiterzuleben, und ihrer Freude über die Nachricht der Schwangerschaft wie auch der damit verknüpften Hoffnung. Andererseits ist mit dem Motiv auch die Rettung Sieglindes und damit Siegfrieds durch Brünnhilde verbunden. Siegfried ist das "hehrste Wunder", das besungen wird und damit Träger einer Zukunftsutopie - einer neuen Chance. Diese Bedeutungen eins zu eins auf die Götterdämmerung zu übertragen, würde jedoch einer Simplifizierung gleichkommen, der auch die musikalisch differierende Gestaltung des Motivs in Walküre und Götterdämmerung widerspricht. Entgegen dem mit vollem Orchester im forte beziehungsweise fortissimo instrumentierten Freudenausbruch Sieglindes in der Walküre erklingt das Motiv am Ende der Götterdämmerung lediglich in Violinen und Flöten im piano. Entsprechend relativiert sich der Zukunftsoptimismus, der in der Walküre noch geradezu euphorisch anmutete. Auch die Tonartensymbolik bringt wenig Licht in die Bedeutung des Schlusses der Götterdämmerung. Das Erlösungsmotiv der letzten Takte erklingt mit Des-Dur in eben jener Tonart, die in der gesamten Tetralogie den Göttern zugeordnet ist - eine Perspektive des Neuen fehlt folglich auch hier. Dass eine derart offene inhaltliche und musikalische Gestaltung eine szenische Interpretation gerade herausfordert, ist offensichtlich. Wie eine solche aussehen kann, werden zwei Beispiele zeigen.

$$
-12-
$$

\section{a) Die Inszenierung von Joachim Herz}

Joachim Herz (Regie), Rudolf Heinrich und Reinhard Heinrich (Bühne und Kostüme) inszenierten den Ring 1973-1976 ${ }^{8}$ in Leipzig als "Klassenkampftragödie" ${ }^{9}$, denn Richard Wagner habe diese "Klassenkampftragödie des 19. Jahrhunderts nun verfremdet, eine parabelhafte Form dafür gewählt und als verfremdetes Kostüm die nordische Mythologie darüber gestülpt ${ }^{\prime 10}$. Die Konsequenz daraus wird gezogen, indem der Ring in die historisch konkrete Zeit des 19. Jahrhunderts verlegt wird - entsprechend sind

\footnotetext{
Für die Beschreibung dieser Inszenierung, die weder als Video- noch als Fernsehaufzeichnung vorliegt, wurde auf Fotos, Programmhefte und Dokumentationsbände zurückgegriffen. Joachim Herz, "Das Rheingold", 1973, in: ders., Theater - Kunst des erfüllten Augenblicks. Briefe, Vorträge, Notate, Gespräche, Essays, hg. von Ilse Kobán, Berlin 1989, S. 159.

10 Joachim Herz, Zur Konzeption des Nibelungenrings, in: Joachim Herz inszeniert Richard Wagners "Ring des Nibelungen" am Opernhaus Leipzig, I. Teil: Das Rheingold/Die Walküre, hg. von der Akademie der Künste der DDR, Berlin 1975 (Arbeitsheft 21), S. $29 \mathrm{f}$.
} 
Bühne und Kostüme gestaltet: etwa die Burg Walhall als Montage aus "Justizpalast, Kaisertreppe, Germania und Eisenkuppel"11 ${ }^{11}$ oder Nibelheim als organisiertes Industrieunternehmen.

$$
-13-
$$

Die letzte Szene der Götterdämmerung ${ }^{12}$ ist optisch bestimmt von der Halle der Gibichungen, einer mit dicken Steinsäulen versehenen Baustruktur. Die Leiche Siegfrieds liegt in der hinteren Bühnenmitte. Als Brünnhilde den "Brand in Walhalls prangende Burg" wirft, stürzt die Gibichungenhalle zusammen, und die große Kuppel der Burg Walhall erscheint dahinter. Die Gibichungen ziehen sich zurück, während sich der Brand (in Form einer choreographisch geführten Feuertanzgruppe) ausbreitet. Nachdem die Rheintöchter den Ring wiedergewinnen, verwandelt sich dieser zurück in das Rheingold, mit dem die Rheintöchter auf Gondeln nach oben entschweben. Die Gibichungen kommen wieder auf die Bühne, während Walhall zu sinken beginnt - hinter der Burg schließt ein schwarzer Decker ${ }^{13}$ die Szene ab. Als Walhall ganz versunken ist, werden weiße Tücher aufgezogen und die nun völlig leere Bühne ist in weißes Licht getaucht. Während die Gibichungen erstaunt ihre neue Umgebung erblicken, beendet ein heller Vorhang die Szene.

$$
-14
$$

Joachim Herz ist der Ansicht, dass der "Schluß [der Götterdämmerung] das Ende einer Welt ist, nicht Ende der Welt"14. Entsprechend fällt seine szenische Gestaltung aus. Einerseits wird der Ring am Ende in das Rheingold zurückverwandelt und existiert damit nicht mehr als Machtsymbol, andererseits symbolisiert die am Ende stehende Helligkeit des Bildes die "Zukunft als Keim"15 oder eine "Tabula Rasa", die Herz so erklärt: "Das Alte ist ausgewischt. Nun fängt ein Neues an. Wie dieses Neue beschaffen sein soll, ist an dieser Stelle nicht zu zeigen. Wagner wusste es nicht. Brünnhildes Rede ist

11 Rudolf Heinrich, In verständlichen Bildern, in: Das Rheingold. Programmheft Opernhaus Leipzig, Leipzig 1973, S. 18.

12 Abbildungen zur letzen Szene der Götterdämmerung finden sich in: Joachim Herz inszeniert Richard Wagners "Ring des Nibelungen" am Opernhaus Leipzig, II. Teil: Siegfried/Götterdämmerung, hg. von der Akademie der Künste der DDR, Berlin 1980 (Arbeitsheft 29), S. 34-37. "Decker" bezeichnet einen von oben kommenden, glatten, meist aus Samt bestehenden Vorhang, der auf der Bühne ein Bild nach hinten abschließen kann, aber auch als Schallvorhang für Umbauten oder als Hauptvorhang eingesetzt wird.

14 Joachim Herz, Mythos auf der Bühne?, in: ders., Theater - Kunst des erfüllten Augenblicks. Briefe, Vorträge, Notate, Gespräche, Essays, hg. von Ilse Kobán, Berlin 1989, S. 205. 
die erste von vielen Reden, die gehalten werden müssen, um die richtigen Worte an die neuen weißen Tafeln zu schreiben." 16

$$
-15-
$$

\section{b) Die Inszenierung von Ruth Berghaus}

Ruth Berghaus (Regie) und Axel Manthey (Bühne und Kostüme) gehen in ihrer Inszenierung (Frankfurt 1985-1987) ${ }^{17}$ den Weg einer Stilisierung, indem sie ein dichtes Netz szenischer Metaphern zwischen Erscheinungs- und Bedeutungsebene spannen, wodurch zur musikalischen Struktur ein komplexes theatralisch-szenisches Analogon entsteht. So spielen im Bühnenbild geometrische Formen eine große Rolle, etwa Kreisund Ringformen, die als Bilder für Totalität und Kontinuität, aber auch für Begrenztheit und Gefangensein fungieren. Auf abstrakter Ebene werden mit dieser Bildlichkeit verschiedene Zusammenhänge sichtbar gemacht.

$$
-16-
$$

Die szenische Gestaltung des Schlusses der Götterdämmerung ${ }^{18}$ gerät bei Ruth Berghaus zu einer Übereinanderschichtung verschiedener Erzählebenen. Die Verwendung der Drehbühne ist dabei der entscheidende Gestaltungsfaktor. Während der gesamten Götterdämmerung ist der Bühnenraum in drei Handlungsräume unterteilt. Auf einer Hälfte der kreisrunden Drehbühne befindet sich der Walkürenfelsen (eine kegelförmige Erhöhung), auf der anderen Hälfte die Gibichungenhalle (ein kahler weißer Raum mit kargem Mobiliar und einem Fernrohr). Die Trennung der beiden Kreishälften besteht jedoch nicht bloß aus einer Mauer, sondern aus einem etwa zwei Meter breiten Zwischenstück, das den Rhein bildet und durch zwei Wände von Walkürenfelsen und Gibichungenhalle getrennt ist. Eine Verbindung der Bühnenräume besteht nur durch eine Tür in der Mitte der Drehbühne. Dieser unterteilte Bühnenraum öffnet sich nun am Ende der Götterdämmerung, indem die Zwischenwände nach oben gefahren werden. Die Halle der Gibichungen gibt den Blick frei auf den mythischen Ort des

\footnotetext{
16 Joachim Herz, Götterdämmerung, in: Joachim Herz inszeniert Richard Wagners "Ring des Nibelungen", II. Teil: Siegfried/Götterdämmerung, hg. von der Akademie der Künste der DDR, Berlin 1980 (Arbeitsheft 29), S. 30.

17 Die Beschreibung dieser Inszenierung folgt einer Video-Aufzeichnung des Opernhauses Frankfurt, die von Maxim Dessau dankenswerterweise zur Verfügung gestellt wurde.

18 Abbildungen zur Inszenierung von Ruth Berghaus finden sich im Bildband Richard Wagner. Der Ring des Nibelungen. Ein Bühnenfestspiel für drei Tage und einen Vorabend. Oper Frankfurt 1985-1987. Fotos von Mara Eggert, hg. von der Oper Frankfurt/Dramaturgie, Frankfurt $1986 / 1987$.
} 
Walkürenfelsens, wie auch die beiden Erzählstränge der Siegfriedhandlung und des Göttermythos am Schluss der Götterdämmerung zusammengeführt werden. Am Beginn des Orchesternachspiels ist der Walkürenfelsen im Vordergrund zu sehen. Durch eine Handbewegung entzündet Brünnhilde einen Feuerkranz auf dem Walkürenfelsen (es ist derselbe Feuerkranz, mit dem sie am Ende der Walküre von Wotan umgeben worden war) und setzt sich auf das Pferd Grane, das seitlich des Walkürenfelsens liegt. Hagen, Gutrune, die Rheintöchter und einige Gibichungen befinden sich im Bühnenhintergrund. Brünnhilde geht zum Feuerkranz, und die Rheintöchter kommen zu ihr. Danach wirft Brünnhilde den Ring ins Feuer und rollt die Hinterseite des Walkürenfelsens hinunter. Hagen geht zum Feuerring und streckt die Hand nach dem Ring aus. Nach seinen Worten "Zurück vom Ring" fällt Hagen in den Feuerkranz, und die Rheintöchter heben den Ring auf. Die Bühne dreht sodann in die Gibichungenhalle zurück. Alle Protagonistinnen und Protagonisten stehen nun verteilt im hinteren Drittel des Bühnenraums, nur Brünnhilde und Gutrune Rücken an Rücken in der Mitte, wobei Brünnhilde den Blick zum Walkürenfelsen gerichtet hat und Gutrune zur Gibichungenhalle. Im Hintergrund wird eine rote Ziegelwand, die das brennende Walhall darstellt, sichtbar. Brünnhilde löst sich von Gutrune und geht zur roten Ziegelwand. Die beiden Trennwände senken sich langsam wieder und verdecken die Beteiligten, die alle in der Bühnenhälfte des Walkürenfelsens geblieben sind. In der Gibichungenhalle liegt nur der tote Gunter wie zuvor auf einem Sessel. Gutrune kommt während der letzten Takte von hinten durch die Tür, geht zum Fernrohr, das in der Mitte positioniert ist, setzt sich auf einen Stuhl und blickt durch das Fernrohr.

$$
-17-
$$

In einer Radiodiskussion sagte Ruth Berghaus: "Am Schluß war mir doch wichtig zu zeigen, daß die Ordnung, die alte Ordnung eigentlich bleibt und zwar in größerer Perfektion. Das Fernrohr wird jetzt benutzt und man guckt, einäugig mittels Technik ins All."19 Der einäugige Blick ist eine der wichtigsten szenischen Metaphern der RingInszenierung von Berghaus - wobei dieser Blick immer durch Bedeutungsambivalenzen geprägt ist. Wotans Einäugigkeit hat ihm einerseits Macht und Wissen beschert, andererseits ist Sehen für ihn nur noch zweidimensional möglich. In der Walküre wird der einäugige Blick zum Erkennungszeichen für die Nachkommen Wotans, Siegmund und

19 Ruth Berghaus in einer Radiodiskussion Ring Gespräch I - Rias I vom Juli 1987, aufgezeichnet von Maxim Dessau, Ruth Berghaus-Archiv, Zeuthen, S. 45. Maxim Dessau sei herzlich für die Erlaubnis zur Sichtung des Nachlasses von Ruth Berghaus gedankt. 
Sieglinde, und führt hier zum einen zur Annäherung der beiden. Zum anderen ist die Einäugigkeit aber auch Zeichen für den illusionistischen Blick auf ein Liebesobjekt. Am Ende der Götterdämmerung kann durch das Fernrohr wieder nur durch ein Auge gesehen werden - auch hier hinterlässt die Metapher zwiespältige Eindrücke. In mancher Hinsicht eröffnet der Blick durch das Fernrohr Perspektiven, die mit freiem Auge niemals erkennbar wären, dagegen bleibt aber das Bild ein zweidimensionales und vermittelt den irrigen Eindruck, dass weit Entferntes in erreichbarer Nähe liegt. Erwähnenswert scheint, dass das Fernrohr in der Götterdämmerung immer ins Bühneninnere zeigt und Gutrunes Blick durch das Fernrohr am Ende der Götterdämmerung der erste und einzige ist, der sich in den Zuschauerraum richtet.

$$
-18-
$$

\section{c) Szenische Lösungen und musikwissenschaftliche Folgerungen}

Sind die eingangs erörterten Fragen angesichts des Schlusses der Götterdämmerung nun in den Inszenierungen von Herz und Berghaus durch konkrete Bilder beantwortet worden - und wenn ja, wie sehen diese szenischen Lösungen aus? Wenn am Ende der Götterdämmerung die Frage nach der Zukunft (wie oben argumentiert) vorherrschend ist, so muss es zu den neuralgischen Punkten einer Inszenierung gehören, positive oder negative Utopien sowie Zukunftsträgerinnen und -träger auszumachen. Die Lösungen hierfür sind bei Herz und Berghaus sehr unterschiedlich ausgefallen.

$$
-19-
$$

Joachim Herz deutet in seiner Inszenierung jene positiven politischen Utopien an, die Richard Wagner in seinen Schriften (nicht aber am Schluss der Götterdämmerung) formuliert hat. Herz transportiert so auf dem Theater die sozialrevolutionären Ansichten Wagners mit. In deren Zentrum - formuliert vor allem in den Zürcher Kunstschriften steht eine Polarität zwischen der damaligen, für Wagner inakzeptablen, von Institutionen geprägten Industriegesellschaft und einer zukünftigen harmonisierenden, selbst organisierten Gesellschaft, als deren Modell die griechische Polis fungiert ${ }^{20}$. Herz betont mit der in weißes Licht getauchten Bühne und den versammelten Gibichungen vor allem zwei Aspekte von Wagners politischen Ansichten. Zum einen ist die weiße Bühne als sichtbares Zeichen für einen völligen Neuanfang zu verstehen, von dem Wagner

\footnotetext{
20 Vgl. Udo Bermbach, Der Wahn des Gesamtkunstwerks. Richard Wagners politisch-ästhetische Utopie, Frankfurt 1994, S. 146-153.
} 
immer wieder spricht. Zum anderen steht das Volk im Mittelpunkt von Herz' Zukunftsvision - jenes Volk, das der Kern von Wagners Darstellung der griechischen Polis ist und die Grundlage seiner gesellschaftspolitischen Utopien. In Wagners Schrift Das Künstlertum der Zukunft etwa heißt es:

"[...] das Volk, das - unbewußt - und deshalb aber eben aus Naturtrieb handelt, wird das Neue zustande bringen; die Kraft des Volkes ist aber eben noch so lange gelähmt, als es von einer veralteten Intelligenz, von einem hemmenden Bewußtsein sich fesseln und leiten lässt: erst wenn diese vollständig von und in ihm vernichtet sind, erst wenn wir alle wissen und begreifen, dass wir nicht unserer Intelligenz, sondern der Notwendigkeit der Natur uns überlassen müssen, wenn wir also so kühn geworden sind, unsere Intelligenz zu verneinen, erhalten wir alle die Kraft aus natürlichem Unbewußtsein, aus der Not heraus das Neue zu produzieren $[\ldots]^{\prime \prime 21}$

Das unbewusst handelnde Volk ist für Wagner das revolutionäre Potenzial seiner Utopien - es wird auch von Joachim Herz in seiner Inszenierung an den Schluss gestellt. Aufgrund dieser positiven Utopie Herz' wird das "Liebeserlösungsmotiv" am Ende der Götterdämmerung in derselben Weise gedeutet, wie es im dritten Akt der Walküre erstmals erscheint - als große Hoffnung. War es dort der noch ungeborene Siegfried, der als Träger einer zukünftigen Veränderung von Sieglinde besungen wurde, so sind es am Ende der Götterdämmerung bei Herz die übrig gebliebenen Menschen, denen das "Liebeserlösungsmotiv" gilt und denen ein Neuanfang gelingen kann.

$-20-$

Ruth Berghaus' szenische Variante des Schlusses der Götterdämmerung erscheint ungleich skeptischer als diejenige von Joachim Herz. Gutrune bleibt - abgesehen vom toten Gunther - als einzige in der leeren Gibichungenhalle zurück und blickt durch ein Fernrohr in Richtung des Zuschauerraumes. Der Blick in die Ferne stellt dabei eine Suche nach Zukunft dar - es ist der Blick auf andere Welten, in denen das Leben vielleicht noch lebenswert erscheint. Gutrune kehrt sich ab von ihrer Welt. Die zerstörte Welt der Gibichungen will sie nicht mehr sehen - ein Signum dafür, dass Berghaus

21 Richard Wagner, Das Künstlertum der Zukunft (1849), in: ders., Dichtungen und Schriften. Jubiläumsausgabe in zehn Bänden, Bd. 5, hg. von Dieter Borchmeyer, Frankfurt 1983, S. 244. 
dieser Welt keine Zukunft mehr gibt. Dass das Ende der Götterdämmerung mit Gutrune von einer Figur bestimmt wird, die in der gesamten Tetralogie kaum präsent oder bedeutend ist, scheint vorderhand wenig überzeugend, wird jedoch aufschlussreicher, wenn der Blick auf die inhaltlichen Positionen der Frauengestalten im Ring gelenkt wird. Die Handlung des Ring findet in einer durch geschlechtliche Polaritäten gekennzeichneten Ordnung statt. Die Macht wird unter Männern wie Wotan, Alberich, Fasolt, Fafner oder Siegfied und deren Handlangern ausgehandelt (bzw. eben nicht ausgehandelt sondern erzwungen). Es sind die Frauen im Ring, die gegen diese Ordnung opponieren: Erda prophezeit in Rheingold Wotan den Untergang und kritisiert in Siegfried sein doppeltes Spiel ${ }^{22}$, Sieglinde bricht in der Walküre aus ihrer bürgerlichen Ehe mit Hunding aus, und Brünnhilde widersetzt sich ihrem Vater Wotan, indem sie Siegmund schützt und Sieglinde zur Flucht verhilft. Brünnhilde ist es schließlich auch, die das Ende der patriarchalischen Weltordnung durch ihren Ritt ins Feuer besiegelt. Gutrune hingegen ist vorerst nur eine Mitläuferin - sie spielt das Machtspiel mit und nimmt erst am Ende wahr, dass sie benutzt worden ist - eine kleine Schachfigur im großen Weltendrama. "In dem Thema - Macht und Liebe bedingen einander - steckt die Eigentumsfrage. Wer ist mein Mann, mein Kind, meine Frau. Wer ist wessen Eigentum. Ein Gedanke dabei ist, wenn diese Eigentumsfrage weg ist, kann eine Möglichkeit der Versöhnung zwischen Gutrune und Brünnhilde utopisch dasein" ${ }^{23}$. Ruth Berghaus spielt mit dieser Aussage auf jenen Moment an, in welchem Gutrune und Brünnhilde Rücken an Rücken stehen - es ist ein Moment der Erkenntnis, in dem sich Brünnhildes Denken in weltverändernden Dimensionen auf Gutrune zu übertragen scheint. Eben deshalb kann eine Fokussierung Gutrunes am Ende der Götterdämmerung konsequent sein. Gutrune ist eine Frau, die in der patriarchalischen Ordnung des Ring unterdrückt worden ist. Indem sie dies erkennt und indem sie die Position Brünnhildes und die weitreichenden Konsequenzen des Geschehens reflektiert, hat sie am Ende der Götterdämmerung vielleicht die Möglichkeit, neue Wege zu gehen.

$22 \quad$ Die Textstelle in der ersten Szene des dritten Aktes lautet: "Der den Trotz lehrte straft den Trotz? Der die Tat entzündet zürnt die Tat? Der die Rechte wahrt, der die Eide hütet, wehret dem Recht, herrscht durch Meineid?" (Richard Wagner, Der Ring des Nibelungen. Ein Bühnenfestspiel für drei Tage und einen Vorabend. Zweiter Tag: Siegfried, in: ders., Dichtungen und Schriften. Jubiläumsausgabe in zehn Bänden, Bd. 3, hg. von Dieter Borchmeyer, Frankfurt 1983, S. 220f.).

23 Ruth Berghaus in einem Gespräch zur Ring-Konzeption an der Universität Mainz am 26. Juni 1987, in: Sigrid Neef, Das Theater der Ruth Berghaus, Berlin 1989, S. 160. 


\section{d) Zeitgeist und Musiktheater}

Wenn Nora Eckert schreibt: "Die Tendenz der Opernregie im 20. Jahrhundert ist klar: Sie will die Werke als Echo des Menschen vorführen, was sich mit der Ansicht des Romantikers Novalis deckt, wonach das Theaterspiel die tätige Reflexion des Menschen über sich selbst ist" ${ }^{24}$, dann spricht sie damit an, was bereits am Beginn dieser Ausführungen angedeutet wurde - dass Inszenierungen durch aktuelle philosophische, politische oder gesellschaftliche Befindlichkeiten beeinflusst sind beziehungsweise diese aufgreifen. Mit Herz' Inszenierung aus den siebziger Jahren des 20. Jahrhunderts und Berghaus' Inszenierung aus den Achtzigern liegen zwei Inszenierungen vor, die einen jeweils vorherrschenden Zeitgeist widerspiegeln.

$$
-22-
$$

Herz' Inszenierung speist sich vor allem aus jenem sozialistischen und neomarxistischen Gedankengut, das in den siebziger Jahren den ideologischen Hintergrund der DDR bildete. Der Ring, als das zentrale Element aller Machtkämpfe in der Tetralogie, steht symbolisch für das Kapital als Angelpunkt der industriellen Ordnung des 19. Jahrhunderts. Der Untergang jener kapitalistischen Ordnung und die Beschwörung einer neuen Gesellschaftsordnung im Sinne einer klassenlosen Gesellschaft sind die Kernpunkte von Herz' Interpretation des Schlusses der Götterdämmerung. Er betont auch, dass er die anti-kapitalistischen, nicht komponierten Schlussverse "Nicht Gut, nicht Gold..." ${ }^{25}$ für den eigentlichen Schluss des Werkes hält, woraus sich auch seine Interpretation des "Liebeserlösungsmotivs" am Ende der Götterdämmerung erklärt:

\footnotetext{
$24 \quad$ Nora Eckert, Von der Oper zum Musiktheater. Wegbereiter und Regisseure, Berlin 1995, S. 10.

25 Richard Wagner, Dichtungen und Schriften. Jubiläumsausgabe in zehn Bänden, Bd. 3, hg. von Dieter Borchmeyer, Frankfurt 1983, S. 313f. Die gesamte, im Privatdruck 1853 formulierte Textstelle, die Wagner 1865 wieder verwarf, lautet: "Nicht Gut, nicht Gold, noch göttliche Pracht; nicht Haus, nicht Hof, noch herrischer Prunk; nicht trüber Verträge trügender Bund, nicht heuchelnder Sitte hartes Gesetz: selig in Lust und Leid lässt - die Liebe nur sein." Hans Mayer nannte diesen Schluss den "Feuerbach-Schluss", um die Beeinflussung Wagners durch den Philosophen terminologisch kenntlich zu machen (vgl. Hans Mayer, Richard Wagners geistige Entwicklung, Düsseldorf u.a. 1954, S. 41).
} 
"Daß dieses Motiv im Grunde ein Feuerbach-Motiv ist, geht aus der Situation hervor, in der es uns im Nibelungenring begegnet, nämlich als Siegfrieds Kommen vorausgesagt wird. [...] hier wird eine Gegenkraft gegen Wotan beschworen. [...] Vom Ursprung her war für Wagner der Nibelungenring die Geschichte von einer alten Ordnung, einem überlebten Gesetz, und unter Schmerzen und Tragik wird die neue Menschheit geboren. Das war seine Grundkonzeption, und so hat er's auch zu Ende geführt. [...] Die zwar nicht komponierten Feuerbach-Verse liegen dem ganzen Werk nachweisbar als poetisch-musikalische Idee zugrunde."26

Herz negiert damit, dass die musikalische Substanz des Motivs - wie oben ausgeführt in Walküre und Götterdämmerung eine andere ist. Zugunsten einer interpretatorischen Aussage setzt er sich über diesen Umstand hinweg. Das Ende der Götterdämmerung ist hier die theatralische Verwirklichung einer neuen, anti-kapitalistischen Gesellschaftsordnung, wie sie vielleicht von Wagner, von Marx, von Herz oder den sozialistischen Staaten erträumt wurde.

$$
-23-
$$

Joachim Herz ist mit dieser positiven politischen Utopie in den siebziger Jahren nicht alleine - auch Patrice Chéreau deutet in seiner Bayreuther Inszenierung von 1976 eine Utopie an, in der Kapitalismuskritik und Emanzipation des Volkes im Mittelpunkt stehen $^{27}$. Solcher Optimismus ist in Inszenierungen der achtziger Jahre des zwanzigsten Jahrhunderts kaum noch vorhanden - vielmehr bestimmen Endzeitphänomene, Machtlosigkeit gegenüber Veränderungen oder Bilder ökologischer Katastrophen den Schluss der Götterdämmerung ${ }^{28}$. Auch Ruth Berghaus' Inszenierung, die zu den bedeutendsten szenischen Realisierungen der achtziger Jahre zählt, ist von diesen Tendenzen beeinflusst. Dass die leere trostlose Halle der Gibichungen, in die Gutrune zurückkehrt, für sie kein Zuhause mehr sein kann, verdeutlicht Gutrunes Blick durch das Fernrohr nach

\footnotetext{
26 Joachim Herz, Zur Konzeption des Nibelungenrings. Die Walküre, in: Joachim Herz inszeniert den "Ring des Nibelungen" am Opernhaus Leipzig, I. Teil: Das Rheingold/Die Walküre, hg. von der Akademie der Künste der DDR, Berlin 1980 (Arbeitsheft 29), S. 61.

27 Zwar spricht Chéreau hinsichtlich der in seiner Inszenierung am Ende in den Orchestergraben blickenden Gibichungen von einer "Welt der Unsicherheit", an anderer Stelle meint er jedoch, "die Werte der Welt müssen wiederaufgebaut und wiedergefunden werden" und scheint damit eine positive Zukunftsutopie anzudeuten. (Patrice Chéreau, Ausführungen im Anhang, in: Pierre Boulez u.a., Der "Ring" Bayreuth 1976-1980, München 1988, S. 120.)

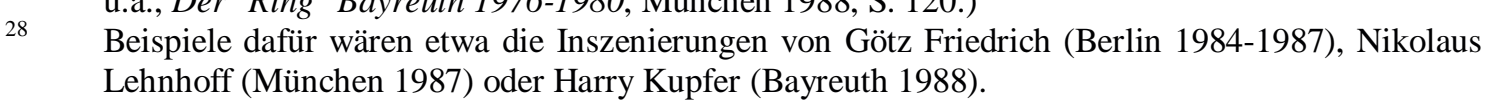


außen. Sie will die Welt der Gibichungen verlassen, aber ob die Ausschau nach Alternativen tatsächlich neue Möglichkeiten bietet, bleibt in der Inszenierung offen.

$$
-24-
$$

Doch Ruth Berghaus' Inszenierung reiht sich nicht bloß in allgemeine Inszenierungswege der achtziger Jahre ein, sondern verfolgt gerade mit der szenischen Lösung für den Schluss der Götterdämmerung einen explizit feministischen Ansatz. Die Denkansätze der sogenannten "Zweiten Frauenbewegung" der siebziger und achtziger Jahre, die sich besonders mit der Befreiung der Frau aus ihrer Unterdrückung durch patriarchalische Machtstrukturen beschäftigt hat, werden von Berghaus durchaus aufgeworfen. Gutrune, die die Entwicklungen der zerstörerischen patriarchalischen Verhaltensweisen der Gibichungen am eigenen Leib erfahren und ihr Fehlverhalten erkannt hat, kann unter Umständen zu einer neuen Lebensart finden.

$$
-25-
$$

\section{Was Forschung und Theater füreinander tun können}

Werden die Realisierungen des Schlusses der Götterdämmerung durch Joachim Herz und Ruth Berghaus als Ganzes betrachtet, so kann zu zweierlei Erkenntnis gelangt werden. Zum ersten sind die beiden Lösungsansätze völlig unterschiedlich und (zumindest was Berghaus betrifft) wenig nahe liegend. Zum zweiten sind aber beide auflösbar. Was die Musiktheaterforschung leisten kann, ist genau jenes Auflösen zunächst vielleicht rätselhaft anmutender Inszenierungsideen. Durch musikdramaturgische Überlegungen, inhaltliche Analysen und historische Kontextualisierungen können Zusammenhänge dargestellt werden, die unterschiedliche szenische Ansätze nachvollziehbar und schlüssig machen. Musiktheaterforschung, verstanden als Forschung zum aktuellen Musiktheater - das heißt nicht als historische Forschung - interdisziplinär zu betreiben besagt, genau diese Zusammenhänge zu stiften, und zwar nicht, weil "Regietheater" sui generis erklärungsbedürftig wäre, sondern weil Voreingenommenheit, die durch starre Erwartungshaltungen und Vorurteile entsteht, bei den Rezipierenden oft ein Weiterdenken dieser Ansätze verhindert.

$$
-26-
$$

Ziel der Musiktheaterforschung wäre es demnach, eine Vermittlungsebene zwischen der wissenschaftlichen Forschung - bei der immer Leerstellen im Hinblick auf nicht eindeutig entscheidbare Fragen bleiben - und den jeweiligen inszenatorischen Lösungen zu 
schaffen. Der Erkenntnisgewinn liegt in der Plausibilisierung eines aktuellen interpretatorischen Ansatzes, aber auch in dessen Rückwirkungen auf die Wissenschaft.

$$
-27-
$$

Dass diese Rückwirkungen implizit bereits existieren, davon zeugen sowohl die Inszenierung von Joachim Herz als auch diejenige von Ruth Berghaus. Wenn gerade Anfang der achtziger Jahre der "linke" Wagner in der musikwissenschaftlichen Forschung en

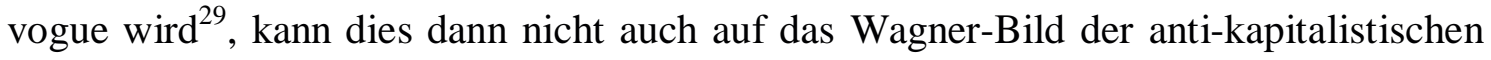
Inszenierungen der siebziger Jahre zurückgeführt werden? Ebenso muss im Falle von Berghaus festgehalten werden, dass die musikwissenschaftliche Auseinandersetzung mit Wagners "Weib der Zukunft" ${ }^{30}$ im Sinne feministischer Utopien erst in der zweiten Hälfte der achtziger Jahre des zwanzigsten Jahrhunderts beginnt. Wenn Susanne Vill in einer der jüngsten Publikationen zu Wagners Frauenfiguren zur Feststellung gelangt: "Trotz der aktiver gestalteten männlichen Protagonisten, erweisen sich in den meisten Musikdramen Wagners die Frauen als entscheidende Handlungsträger" ${ }^{31}$ und sie dies mit vorhergehenden Untersuchungen zur Frau als Opfer in der Oper kontrastiert ${ }^{32}$, schlägt sie damit genau in jene Kerbe, in der Berghaus ihre Inszenierung positioniert. Auch da scheint die Wissenschaft eben jene Wege zu beschreiten, die das zeitgenössische Regietheater zur Diskussion gestellt hat. Es wäre nicht abwegig, sondern vielmehr besonders erstrebenswert, in der Wissenschaft eine Hellhörigkeit gegenüber diesen Tendenzen an den Tag zu legen, ihnen nachzuspüren und mit den angemessenen Methoden einer Musiktheaterforschung differenziert zu begegnen.

\footnotetext{
29 Vgl. beispielsweise Martin Gregor-Dellin, Richard Wagner. Sein Leben · Sein Werk · Sein Jahrhundert, München 1980; Franz-Peter Opelt, Richard Wagner - Revolutionär oder Staatsmusikant?, Frankfurt u.a. 1987; Udo Bermbach (Hg.), In den Trümmern der eignen Welt. Richard Wagners "Der Ring des Nibelungen", Berlin u.a. 1989; ders., Der Wahn des Gesamtkunstwerks. Richard Wagners politisch-ästhetische Utopie, Frankfurt 1994. Richard Wagner, Bemerkungen zur Aufführung der Oper "Der fliegende Holländer" (1852), in: ders., Dichtungen und Schriften. Jubiläumsausgabe in zehn Bänden, Bd. 2, hg. von Dieter Borchmeyer, Frankfurt 1983, S. 50. Susanne Vill, "Das Weib der Zukunft". Frauen und Frauenstimmen bei Wagner, in: "Das Weib der Zukunft". Frauengestalten und Frauenstimmen bei Richard Wagner, hg. von Susanne Vill, Stuttgart 2000, S. 12.

32 Vill nennt hier vor allem den "Klassiker" von Catherine Clément (L'opéra ou la défaite des femmes, Paris 1979).
} 


\section{Die andere Seite der Medaille}

Wenn die Feststellung berechtigt ist, dass die Musiktheaterforschung neue Impulse aus den inszenatorischen Ansätzen von Opernaufführungen gewinnt, so stellt sich die Frage, ob dies auch umgekehrt gelten kann - ob also die Musiktheaterforschung der Musiktheaterpraxis Anregungen gibt. Mike Ashman schreibt in seinem Aufsatz Producing Wagner:

"In the 1970s a flood of newly available background material - 'Cosima Wagner's Diaries', the 'Brown Book', a complete edition of Wagner's autobiography, 'Mein Leben' - added more fuel to the dramaturgical interest in the works. The focus shifted to exploring the works in the context of their contemporary history, both Wagner's and that of the world around him." 33

Geht aus dieser Äußerung Ashmans einerseits hervor, dass es musikwissenschaftliche Einflüsse auf die Musiktheaterpraxis gibt, so lässt sie andererseits durchscheinen, dass diese Einflüsse sich wohl auf neu zugängliches Quellenmaterial beschränken. Ist die Musiktheaterforschung für das Theater damit nur als Quellenforschung interessant?

$-29-$

Wenn in dem vorliegenden Aufsatz an anderer Stelle nach einem spezifischen Zeitgeist gefragt wurde, der den beiden besprochenen Inszenierungen zugrunde liegt, dann ist damit nicht nur gemeint, dass szenische Interpretationen die Wahrnehmungsperspektive einer Zeit widerspiegeln. Regietheater entscheidet sich meist ganz bewusst dafür, ein Werk aus einer bestimmten Zeit heraus zu deuten. Viele Regisseurinnen und Regisseure des Ring des Nibelungen äußern sich in diese Richtung ${ }^{34}$, auch Ruth Berghaus, wenn sie

$33 \quad$ Mike Ashman, Producing Wagner, in: Wagner in Performance, hg. von Barry Millington und Stewart Spencer, New Haven u.a. 1992, S. 47.

34 Beispielsweise Götz Friedrich: "Der Ring ist freigegeben worden dafür, das zu sein, was er von Anfang an war und hätte sein sollen: größte Herausforderung an die Ideen und an die lebendige Phantasie derer, die sich mit ihm beschäftigen dürfen; permanenter moderner Mythos, der zu vergegenwärtigen ist und der danach fragt, ob wir das Prinzip Hoffnung noch wollen oder dulden." (Regieprobleme im "Ring", in: In den Trümmern der eignen Welt. Richard Wagners "Der Ring des Nibelungen", hg. von Udo Bermbach, Berlin u.a. 1989, S. 101); Harry Kupfer: "Der Ring ist heute noch genauso aktuell wie zur Zeit seiner Entstehung, vielleicht sogar in einer höhern Art und Weise [...]; das was an Fragen, an Kritik aufgeworfen wird, ist so grundsätzlich, daß es für die Gefährdung in unserem Jahrhundert noch zutreffender ist als für das vergangene Jahrhundert." (Harry Kupfer im Gespräch mit Michael Lewin, in: Michael Lewin, Der Ring. 
meint: "Die Vielschichtigkeit dessen, was an einem Opernabend geschieht, macht heute das Interesse an Oper aus. Sicher hängt das mit unserem Leben zusammen, mit unserem Alltag und der Welt." 35 "Unsere Welt" ist der Nährboden für szenische Interpretationen der gegenwärtigen Theaterlandschaft ${ }^{36}$ - die Musiktheaterforschung scheint für die philologischen Details zuständig zu sein.

$$
-30-
$$

Doch ist das alles, was die Musiktheaterwissenschaft $\mathrm{zu}$ bieten hat? Geht die Opernforschung nicht weit darüber hinaus ${ }^{37}$, und wäre es nicht wert, dass diese Forschung auch in der lebendigen Auseinandersetzung mit Werken auf der Musiktheaterbühne rezipiert wird? Dies ist kein Vorwurf an Regisseurinnen und Regisseure beziehungsweise Dramaturginnen und Dramaturgen, die ihre "Hausaufgaben" nicht gemacht haben (obwohl dieser Vorwurf in manchen Fällen sicher gerechtfertigt wäre), es ist ein Appell an eine Musiktheaterforschung, die hinsichtlich ihrer Positionierung im Musiktheaterbetrieb allenfalls ein Nischendasein führt ${ }^{38}$. Es ist zuvor hinreichend gezeigt worden, dass Regietheater als Forschungsobjekt für die Musikwissenschaft attraktiv ist - der Schritt könnte aber auch in die andere Richtung gesetzt werden, indem die Musiktheaterforschung ihre Erkenntnisgewinne gezielter an das Theater heranträgt.

Bayreuth 1988-1992, Hamburg 1991, S. 92); Nikolaus Lehnhoff: "Warum spielen wir den Ring? Wir spielen ihn, weil er auch von uns erzählt." (Ein Klima der Endzeit: Wagners "Ring" aus unserer Sicht, in: Opernwelt 28/4 [1987], S. 13). Ruth Berghaus in einem Gespräch zur Ring-Konzeption an der Universität Mainz am 26. Juni 1987, in: Sigrid Neef, Das Theater der Ruth Berghaus, Berlin 1989, S. 157.

36 Besonders das Theater der siebziger und achtziger Jahre war dabei geprägt von bildungsbürgerlichen Idealen der Aufklärung, indem in den Inszenierungen durch Aktualisierungstendenzen dem Publikum ein Spiegel vorgehalten werden sollte. Auch wenn dieser Anspruch gegenwärtig eher an Bedeutung verloren hat, generieren sich viele Inszenierungen direkt oder assoziativ aus Bildern der realen Welt (auf eine Aufzählung von Beispielen sei an dieser Stelle verzichtet, da das Phänomen allgegenwärtig ist).

37 Es wäre müßig, hier alle Entwicklungen, Forschungsrichtungen und Methoden der Opernforschung aufzuzählen. Erwähnt seien beispielsweise die Entwicklungen rund um die "New Musicology" oder neuere kultur- und sozialhistorische Forschungen zum Musiktheater. Dass mittlerweile die Musikwissenschaft in den Programmheften verschiedener Theater präsent ist und die von ihr gelieferten Beiträge auch wissenschaftlich anerkannt sind (etwa in den Programmheften der Bayreuther Festspiele oder der Opernhäuser Frankfurt und Stuttgart), ist ein erster Schritt in die richtige Richtung. Kooperationen zwischen Musikwissenschaft und Theater könnten jedoch noch viel weiter gehen. 